\title{
Composition Operators from $p$-Bloch Space to $q$-Bloch Space on the Fourth Cartan-Hartogs Domains
}

\author{
Jianbing Su and Chao Zhang \\ School of Mathematics and Statistics, Jiangsu Normal University, Xuzhou, Jiangsu 221116, China \\ Correspondence should be addressed to Jianbing Su; sujb@jsnu.edu.cn
}

Received 15 June 2015; Revised 22 August 2015; Accepted 17 September 2015

Academic Editor: Ruhan Zhao

Copyright ( 2015 J. Su and C. Zhang. This is an open access article distributed under the Creative Commons Attribution License, which permits unrestricted use, distribution, and reproduction in any medium, provided the original work is properly cited.

We obtain new generalized Hua's inequality corresponding to $Y_{\mathrm{IV}}(N, n ; K)$, where $Y_{\mathrm{IV}}(N, n ; K)$ denotes the fourth Cartan-Hartogs domain in $\mathbb{C}^{N+n}$. Furthermore, we introduce the weighted Bloch spaces on $Y_{\mathrm{IV}}(N, n ; K)$ and apply our inequality to study the boundedness and compactness of composition operator $C_{\phi}$ from $\beta^{p}\left(Y_{\mathrm{IV}}(N, n ; K)\right)$ to $\beta^{q}\left(Y_{\mathrm{IV}}(N, n ; K)\right)$ for $p \geq 0$ and $q \geq 0$.

\section{Introduction}

The study of composition operators on various Banach spaces of analytic functions has been a long active field in complex and functional analysis. The composition operators as well as related operators known as the weighted composition operators between the Bloch space and Lipschitz space were investigated in $[1,2]$ in the case of the unit disk. The study of the composition operators on the Bloch space was given in [3] for the polydisc, in $[4,5]$ for the unit ball, and in [6-9] for the bounded symmetric domains.

In 1930s, all irreducible bounded symmetric domains were divided into six types by E. Cartan. The first four types of irreducible domains are called the classical bounded symmetric domains. The other two types, called exceptional domains, consist of one domain each (a 16- and 27-dimensional domain). In 2000, Yin constructed four kinds of domains corresponding to the classical bounded symmetric domains, called the Cartan-Hartogs domains [10]. It is known that the Cartan-Hartogs domains are nonhomogeneous domains except the unit ball. So it is different from the bounded symmetric domains. The fourth Cartan-Hartogs domains, denoted by $Y_{\mathrm{IV}}(N, n ; K)$, can be expressed as

$$
\begin{aligned}
& Y_{\mathrm{IV}}(N, n ; K):=\left\{w \in \mathbb{C}^{N}, z \in \mathfrak{R}_{\mathrm{IV}}(n):|w|^{2 K}<1\right. \\
& \left.\quad+\left|z z^{T}\right|^{2}-2 \bar{z} z^{T}, K>0\right\},
\end{aligned}
$$

where $\mathfrak{R}_{\mathrm{IV}}(n)=\left\{z: z \in \mathbb{C}^{n}, 1+\left|z z^{T}\right|^{2}-2 \bar{z} z^{T}>\right.$ $\left.0,1-\left|z z^{T}\right|^{2}>0\right\}$ is the fourth classical bounded symmetric domains [11] and $z^{T}$ is the transpose of $z$.

For simplicity, we will write $Y_{\mathrm{IV}}$ for $Y_{\mathrm{IV}}(N, n ; K)$ if no ambiguity can arise.

Let $\phi=\left(\phi_{i}\right)_{N+n}$ be a holomorphic self-map of $Y_{\mathrm{IV}}$. The class of all holomorphic functions on $Y_{\text {IV }}$ is denoted by $H\left(Y_{\text {IV }}\right)$. The composition operator $C_{\phi}$ on $H\left(Y_{\text {IV }}\right)$ is defined by $\left(C_{\phi} f\right)(z, w)=f(\phi(z, w))$ for all $(z, w) \in Y_{\text {IV }}$ and $f \in H\left(Y_{\text {IV }}\right)$.

In 1955, Hua in [12] proved an inequality: if $Z_{1}, Z_{2}$ are $n \times n$ complex matrices and $I-Z_{1} \bar{Z}_{1}^{T}, I-Z_{2} \bar{Z}_{2}^{T}$ are both Hermitian positive definite matrices, then

$$
\begin{aligned}
& \operatorname{det}\left(I-Z_{1}{\overline{Z_{1}}}^{T}\right) \operatorname{det}\left(I-Z_{2}{\overline{Z_{2}}}^{T}\right) \\
& \leq\left|\operatorname{det}\left(I-Z_{1}{\overline{Z_{2}}}^{T}\right)\right|^{2} .
\end{aligned}
$$

Equality holds if and only if $Z_{1}=Z_{2}$.

In 2015, Su et al. obtained generalized Hua's inequality corresponding to the first Cartan-Hartogs domain $Y_{\text {I }}$ (see Theorem 1 in [13]). From Theorem 1 in [13], it is easy to get more precise inequality (see Lemma 4). Furthermore, we obtain new generalized Hua's inequality corresponding to $Y_{\text {IV }}$ (see Lemma 5). 
In this paper, we define the $p$-Bloch space $\beta^{p}\left(Y_{\text {IV }}\right)$ as the space that consists of all $f \in H\left(Y_{\text {IV }}\right)$ such that

$$
\begin{aligned}
& \|f\|_{\beta^{p}} \\
& =|f(0,0)| \\
& \quad+\sup _{(z, w) \in Y_{\text {IV }}}\left(1-2 \bar{z} z^{T}+\left|z z^{T}\right|^{2}-|w|^{2 K}\right)^{p}|\nabla f(z, w)| \\
& <+\infty
\end{aligned}
$$

where

$$
\begin{aligned}
& \nabla f(z, w)=\left(\frac{\partial f(z, w)}{\partial z_{1}}, \ldots, \frac{\partial f(z, w)}{\partial z_{n}}, \frac{\partial f(z, w)}{\partial w_{1}}, \ldots,\right. \\
& \left.\frac{\partial f(z, w)}{\partial w_{N}}\right), \\
& |\nabla f(z, w)|^{2}=\sum_{1 \leq \alpha \leq n}\left|\frac{\partial f(z, w)}{\partial z_{\alpha}}\right|^{2}+\sum_{1 \leq \beta \leq N}\left|\frac{\partial f(z, w)}{\partial w_{\beta}}\right|^{2} .
\end{aligned}
$$

It is clear that $\beta^{p}\left(Y_{\text {IV }}\right)$ is a set of constant functions when $p<0$, so we assume that $p \geq 0$.

In this paper, we will obtain some results about the composition operators for the case of the weighted Bloch space on the fourth Cartan-Hartogs domain. In Section 2, we state several auxiliary results most of which will be used in the proofs of the main results. In Sections 3 and 4, we establish the main results of the paper. We give the sufficient conditions and necessary conditions for the boundedness (in Section 3) and the compactness (in Section 4) of composition operator $C_{\phi}$ from $\beta^{p}\left(Y_{\text {IV }}\right)$ to $\beta^{q}\left(Y_{\text {IV }}\right)$, where $p \geq 0, q \geq 0$.

\section{Some Lemmas}

In order to obtain our main results, we need the following lemmas.

Lemma 1. If

$$
\begin{gathered}
\frac{\left(1+\left|z z^{T}\right|^{2}-2|z|^{2}-|w|^{2 K}\right)^{q}}{\left(1+\left|z_{2} z_{2}^{T}\right|^{2}-2\left|z_{2}\right|^{2}-\left|w_{2}\right|^{2 K}\right)^{p}}\left|\phi^{\prime}(z, w)\right|=O(1), \\
\left((z, w) \in Y_{I V}, \quad\left(z_{2}, w_{2}\right)=\phi(z, w) \longrightarrow \partial Y_{I V}\right),
\end{gathered}
$$

then

$$
\frac{\left(1+\left|z z^{T}\right|^{2}-2|z|^{2}-|w|^{2 K}\right)^{q}}{\left(1+\left|z_{2} z_{2}^{T}\right|^{2}-2\left|z_{2}\right|^{2}-\left|w_{2}\right|^{2 K}\right)^{p}}\left|\phi^{\prime}(z, w)\right|<\infty
$$

for all $(z, w) \in Y_{I V}$ and $\left(z_{2}, w_{2}\right)=\phi(z, w)$. Where $\phi=$ $\left(\phi_{1}, \phi_{2}, \ldots, \phi_{n}, \ldots, \phi_{N+n}\right)$,

$$
\begin{aligned}
& \phi^{\prime}(z, w) \\
& =\left(\begin{array}{cccccc}
\frac{\partial \phi_{1}}{\partial z_{1}} & \cdots & \frac{\partial \phi_{1}}{\partial z_{n}} & \frac{\partial \phi_{1}}{\partial w_{1}} & \cdots & \frac{\partial \phi_{1}}{\partial w_{N}} \\
\vdots & \ddots & \vdots & \vdots & \vdots & \vdots \\
\frac{\partial \phi_{n}}{\partial z_{1}} & \cdots & \frac{\partial \phi_{n}}{\partial z_{n}} & \frac{\partial \phi_{n}}{\partial w_{1}} & \cdots & \frac{\partial \phi_{n}}{\partial w_{N}} \\
\frac{\partial \phi_{n+1}}{\partial z_{1}} & \cdots & \frac{\partial \phi_{n+1}}{\partial z_{n}} & \frac{\partial \phi_{n+1}}{\partial w_{1}} & \cdots & \frac{\partial \phi_{n+1}}{\partial w_{N}} \\
\vdots & \ddots & \vdots & \vdots & \vdots & \vdots \\
\frac{\partial \phi_{n+N}}{\partial z_{1}} & \cdots & \frac{\partial \phi_{n+N}}{\partial z_{n}} & \frac{\partial \phi_{n+N}}{\partial w_{1}} & \cdots & \frac{\partial \phi_{n+N}}{\partial w_{N}}
\end{array}\right), \\
& \left|\phi^{\prime}(z, w)\right|^{2} \\
& =\sum_{1 \leq \alpha \leq n} \sum_{1 \leq k \leq N+n}\left|\frac{\partial \phi_{k}}{\partial z_{\alpha}}\right|^{2}+\sum_{1 \leq \beta \leq N} \sum_{1 \leq k \leq N+n}\left|\frac{\partial \phi_{k}}{\partial w_{\beta}}\right|^{2} .
\end{aligned}
$$

Proof. From (5), there exists a constant $\delta>0$ such that

$$
\frac{\left(1+\left|z z^{T}\right|^{2}-2|z|^{2}-|w|^{2 K}\right)^{q}}{\left(1+\left|z_{2} z_{2}^{T}\right|^{2}-2\left|z_{2}\right|^{2}-\left|w_{2}\right|^{2 K}\right)^{p}}\left|\phi^{\prime}(z, w)\right| \leq C_{1}
$$

whenever $\operatorname{dist}\left(\left(z_{2}, w_{2}\right), \partial Y_{\text {IV }}\right)<\delta$, where $C_{1}$ is a positive number.

Set $E_{\delta}=\left\{\left(z_{2}, w_{2}\right) \in Y_{\mathrm{IV}}: \operatorname{dist}\left(\left(z_{2}, w_{2}\right), \partial Y_{\mathrm{IV}}\right) \geq \delta\right\}$. It is easy to know that $E_{\delta}$ is a compact subset of $Y_{\mathrm{IV}}$. Thus, there exists a constant $M \in(0,1)$ such that $M \leq 1+\left|z_{2} z_{2}^{T}\right|^{2}-2\left|z_{2}\right|^{2}-$ $\left|w_{2}\right|^{2 K} \leq 1$. So

$$
\frac{1}{\left(1+\left|z_{2} z_{2}^{T}\right|^{2}-2\left|z_{2}\right|^{2}-\left|w_{2}\right|^{2 K}\right)^{p}} \leq \frac{1}{M^{p}}<+\infty .
$$

Therefore, there exists a constant $C$ such that

$$
\frac{\left(1+\left|z z^{T}\right|^{2}-2|z|^{2}-|w|^{2 K}\right)^{q}}{\left(1+\left|z_{2} z_{2}^{T}\right|^{2}-2\left|z_{2}\right|^{2}-\left|w_{2}\right|^{2 K}\right)^{p}}\left|\phi^{\prime}(z, w)\right| \leq C
$$

for all $(z, w) \in Y_{\mathrm{IV}}$ and $\left(z_{2}, w_{2}\right)=\phi(z, w)$. The proof is completed.

Lemma 2. Let $H$ be a compact subset of $Y_{I V}$. Then, there exists a constant $C>0$ such that

$$
\int_{0}^{1} \frac{|(z, w)|}{\left(1+t^{4}\left|z z^{T}\right|^{2}-2 t^{2}|z|^{2}-|t w|^{2 K}\right)^{p}} d t<C
$$

for all $(z, w) \in H$. 
Proof. When $p=0$, we have

$$
\begin{aligned}
& \int_{0}^{1} \frac{|(z, w)|}{\left(1+t^{4}\left|z z^{T}\right|^{2}-2 t^{2}|z|^{2}-|t w|^{2 K}\right)^{p}} d t \\
& \quad=\int_{0}^{1}|(z, w)| d t .
\end{aligned}
$$

Since $|w|^{2 K}<1+\left|z z^{T}\right|^{2}-2|z|^{2} \leq 1$ and $|(z, w)|<2$, $\int_{0}^{1}|(z, w)| d t<2$.

When $p>0$, denote $E_{\delta}:=\left\{(z, w) \in Y_{\mathrm{IV}}: 1+\left|z z^{T}\right|^{2}-\right.$ $\left.2|z|^{2}-|w|^{2 K} \geq \delta\right\}, \delta \in(0,1)$. For any compact subset $H \subset$ $Y_{\text {IV }}$, there exists a constant $\delta \in(0,1)$ such that $H \subset E_{\delta}$.

For any $(z, w) \in H, t \in[0,1]$, we have

$$
\begin{aligned}
|t w|^{2 K} & \leq|w|^{2 K} \leq 1+\left|z z^{T}\right|^{2}-2|z|^{2} \\
& \leq 1+t^{4}\left|z z^{T}\right|^{2}-2 t^{2}|z|^{2} .
\end{aligned}
$$

Furthermore,

$$
\begin{aligned}
1+ & t^{4}\left|z z^{T}\right|^{2}-2 t^{2}|z|^{2}-|t w|^{2 K} \\
& \geq 1+\left|z z^{T}\right|^{2}-2|z|^{2}-|w|^{2 K} \geq \delta>0 .
\end{aligned}
$$

Thus,

$$
\begin{aligned}
0 & <\frac{1}{1+t^{4}\left|z z^{T}\right|^{2}-2 t^{2}|z|^{2}-|t w|^{2 K}} \\
& \leq \frac{1}{1+\left|z z^{T}\right|^{2}-2|z|^{2}-|w|^{2 K}} \leq \frac{1}{\delta} .
\end{aligned}
$$

So we have

$$
\int_{0}^{1} \frac{|(z, w)|}{\left(1+t^{4}\left|z z^{T}\right|^{2}-2 t^{2}|z|^{2}-|t w|^{2 K}\right)^{p}} d t<\frac{2}{\delta^{p}}
$$

Letting $C=\max \left\{2,2 / \delta^{p}\right\}$, we can get

$$
\int_{0}^{1} \frac{|(z, w)|}{\left(1+t^{4}\left|z z^{T}\right|^{2}-2 t^{2}|z|^{2}-|t w|^{2 K}\right)^{p}} d t<C
$$

for all $(z, w) \in H$. The proof is completed.

Lemma 3. Let $f \in \beta^{p}\left(Y_{I V}\right)$ and $H$ be a compact subset of $Y_{I V}$. Then, there exists a constant $C>0$ such that

$$
|f(z, w)| \leq C\|f\|_{\beta^{p}} \quad \forall(z, w) \in H .
$$

Proof. By Lemma 2, there exists a constant $C>0$ such that

$$
\begin{aligned}
& |f(z, w)|=\left|f(0,0)+\int_{0}^{1}\langle\nabla f(t z, t w),(\bar{z}, \bar{w})\rangle d t\right| \\
& \leq|f(0,0)|+\int_{0}^{1}|\nabla f(t z, t w)||(\bar{z}, \bar{w})| d t \\
& \leq|f(0,0)| \\
& \quad+\|f\|_{\beta^{p}} \int_{0}^{1} \frac{|(z, w)|}{\left(1+t^{4}\left|z z^{T}\right|^{2}-2 t^{2}|z|^{2}-|t w|^{2 K}\right)^{p}} d t \\
& \leq C\|f\|_{\beta^{p}} .
\end{aligned}
$$

The proof is completed.

Lemma 4. Let $Z_{1}, Z_{2} \in \mathbb{C}^{m \times n}, W_{1}, W_{2} \in \mathbb{C}^{N}$, and $K>0$. If $I_{m}-Z_{1}{\overline{Z_{1}}}^{T}>0, I_{m}-Z_{2}{\overline{Z_{2}}}^{T}>0,\left|W_{1}\right|^{2 K}<\operatorname{det}\left(I_{m}-Z_{1}{\overline{Z_{1}}}^{T}\right)$, and $\left|W_{2}\right|^{2 K}<\operatorname{det}\left(I_{m}-Z_{2} \bar{Z}_{2}^{T}\right)$, then

$$
\begin{aligned}
& {\left[\operatorname{det}\left(I_{m}-Z_{1}{\overline{Z_{1}}}^{T}\right)-\left|W_{1}\right|^{2 K}\right]} \\
& \cdot\left[\operatorname{det}\left(I_{m}-Z_{2}{\overline{Z_{2}}}^{T}\right)-\left|W_{2}\right|^{2 K}\right] \\
& \quad \leq\left[\left|\operatorname{det}\left(I_{m}-Z_{1}{\overline{Z_{2}}}^{T}\right)\right|-\left(\left|W_{1}\right|\left|W_{2}\right|\right)^{K}\right]^{2} .
\end{aligned}
$$

Proof. Set $r_{1}=\left|W_{1}\right|, r_{2}=\left|W_{2}\right|$. Obviously, $r_{1}, r_{2} \in \mathbb{R}$. So we can get (21) by the process of the proof on Theorem 1 in [13].

The following conclusion of Lemma 5 is new generalized Hua's inequality corresponding to $Y_{\mathrm{IV}}$.

Lemma 5. Let $z, u \in \mathfrak{R}_{I V}(n), w, v \in \mathbb{C}^{N}$, and $K>0$. If $1+$ $\left|z z^{T}\right|^{2}-2|z|^{2}-|w|^{2 K}>0$ and $1+\left|u u^{T}\right|^{2}-2|u|^{2}-|v|^{2 K}>0$, then

$$
\left(1+\left|z z^{T}\right|^{2}-2|z|^{2}-|w|^{2 K}\right)
$$

$$
\begin{aligned}
& \cdot\left(1+\left|u u^{T}\right|^{2}-2|u|^{2}-|v|^{2 K}\right) \\
& \leq\left[\left|1+z z^{T} \overline{u u^{T}}-2 z \overline{u^{T}}\right|-(|w||v|)^{K}\right]^{2} .
\end{aligned}
$$

Proof. When $z, u \in \mathfrak{R}_{\mathrm{IV}}(n)$, there exists an orthogonal matrix $\Gamma$ (see [11]) such that

$$
\begin{aligned}
& z=\left(z_{1}^{*}, z_{2}^{*}, z_{3}^{*}, z_{4}^{*}, 0, \ldots, 0\right) \Gamma, \\
& u=\left(u_{1}^{*}, u_{2}^{*}, u_{3}^{*}, u_{4}^{*}, 0, \ldots, 0\right) \Gamma,
\end{aligned}
$$

where $z_{1}^{*}, z_{2}^{*}, z_{3}^{*}, z_{4}^{*}, u_{1}^{*}, u_{2}^{*}, u_{3}^{*}, u_{4}^{*} \in \mathbb{C}$.

Set

$$
\begin{aligned}
& Z=\left(\begin{array}{ll}
z_{1}^{*}+i z_{2}^{*} & z_{1}^{*}-i z_{2}^{*} \\
z_{3}^{*}-i z_{4}^{*} & z_{3}^{*}+i z_{4}^{*}
\end{array}\right), \\
& U=\left(\begin{array}{ll}
u_{1}^{*}+i u_{2}^{*} & u_{1}^{*}-i u_{2}^{*} \\
u_{3}^{*}-i u_{4}^{*} & u_{3}^{*}+i u_{4}^{*}
\end{array}\right) .
\end{aligned}
$$


Then,

$$
\begin{gathered}
1+\left|z z^{T}\right|^{2}-2|z|^{2}=\operatorname{det}\left(I_{2}-Z \overline{Z^{T}}\right)>0, \\
1+\left|u u^{T}\right|^{2}-2|u|^{2}=\operatorname{det}\left(I_{2}-U \overline{U^{T}}\right)>0, \\
1+z z^{T} \overline{u u^{T}}-2 z \overline{u^{T}}=\operatorname{det}\left(I_{2}-\overline{Z^{T}} U\right) \neq 0 .
\end{gathered}
$$

From Lemma 4, we can get

$$
\begin{aligned}
& \left(1+\left|z z^{T}\right|^{2}-2|z|^{2}-|w|^{2 K}\right) \\
& \cdot\left(1+\left|u u^{T}\right|^{2}-2|u|^{2}-|v|^{2 K}\right) \\
& \leq\left[\left|1+z z^{T} \overline{u u^{T}}-2 z \overline{u^{T}}\right|-(|w||v|)^{K}\right]^{2} .
\end{aligned}
$$

The proof is completed.

Lemma 6. The composition operator $C_{\phi}$ from $\beta^{p}\left(Y_{I V}\right)$ to $\beta^{q}\left(Y_{I V}\right)$ is compact if and only if $\left\|C_{\phi} f_{v}\right\|_{\beta^{q}} \rightarrow 0$ as $v \rightarrow \infty$ for every bounded sequence $\left\{f_{v}\right\}$ in $\beta^{p}\left(Y_{I V}\right)$ such that $f_{v} \rightarrow 0$ uniformly on every compact subset of $Y_{I V}$.

Proof. Assume that $C_{\phi}$ from $\beta^{p}\left(Y_{\text {IV }}\right)$ to $\beta^{q}\left(Y_{\text {IV }}\right)$ is compact. Let $\left\{f_{v}\right\}$ be a bounded sequence in $\beta^{p}\left(Y_{\text {IV }}\right)$ such that $f_{v} \rightarrow 0$ uniformly on every compact subset of $Y_{\text {IV }}$. Suppose $\left\|C_{\phi} f_{v}\right\|_{\beta q} \rightarrow 0$ as $v \rightarrow \infty$. Then, there exists a subsequence $\left\{f_{v_{j}}\right\}$ of $\left\{f_{v}\right\}$ such that inf ${ }_{j}\left\|C_{\phi} f_{v_{j}}\right\|_{\beta^{q}}>0$. Since $C_{\phi}$ is compact, there exists a subsequence of the bound subsequence $\left\{f_{v_{j}}\right\}$, still denoted as $\left\{f_{v_{j}}\right\}$, such that $\lim _{j \rightarrow \infty}\left\|f-C_{\phi} f_{v_{j}}\right\|_{\beta^{q}}=0$, $f \in \beta^{q}\left(Y_{\text {IV }}\right)$. For any compact set $H \subset Y_{\text {IV }}$, there exists a constant $C$ depending only on $H$ such that

$$
\begin{aligned}
\left|\left(f-C_{\phi} f_{v_{j}}\right)(z, w)\right| \leq C\left\|f-C_{\phi} f_{v_{j}}\right\|_{\beta^{q}} \longrightarrow & 0, \\
& j \longrightarrow \infty .
\end{aligned}
$$

Thus, $\left\{f-C_{\phi} f_{v_{j}}\right\} \rightarrow 0$ uniformly on compact subset $H$. For $\forall \varepsilon>0$, there exists a constant $J_{1}$ such that

$$
\left|f(z, w)-f_{v_{j}}(\phi(z, w))\right|<\varepsilon
$$

whenever $j>J_{1}$ and $(z, w) \in H$.

Note that $\left\{f_{v_{j}}\right\} \rightarrow 0$ uniformly on compact subset $H$; then, for the above $\varepsilon$, there exists a constant $J_{2}$ such that

$$
\left|f_{v_{j}}(z, w)\right|<\varepsilon
$$

whenever $j>J_{2}$ and $(z, w) \in H$. Let $J=\max \left\{J_{1}, J_{2}\right\}$; from (28) and (29), we get

$$
|f(z, w)|<\left|f_{v_{j}}(\phi(z, w))\right|+\varepsilon<2 \varepsilon
$$

whenever $j>J$ and $(z, w) \in E:=H \cap \phi(H)$. So $f(z, w)=0$; furthermore, $f \equiv 0$ on $Y_{\text {IV }}$. This is a contradiction and we have $\lim _{j \rightarrow \infty}\left\|C_{\phi} f_{v_{j}}\right\|_{\beta^{q}}=0$.
Conversely, let $\left\{f_{v}\right\}$ be a bounded sequence in $\beta^{p}\left(Y_{\text {IV }}\right)$ with $\left\|f_{v}\right\|_{\beta^{p}} \leq C$. Then, there exists a subsequence $\left\{f_{v_{j}}\right\}$ of $\left\{f_{v}\right\}$ and $\left\{f_{v_{j}}\right\} \rightarrow f$ as $j \rightarrow+\infty$. Thus,

$$
\lim _{j \rightarrow \infty}\left\|C_{\phi}\left(f_{v_{j}}-f\right)\right\|_{\beta^{q}}=\lim _{j \rightarrow \infty}\left\|C_{\phi} f_{v_{j}}-C_{\phi} f\right\|_{\beta^{q}}=0 .
$$

Therefore, $C_{\phi}: \beta^{p}\left(Y_{\text {IV }}\right) \rightarrow \beta^{q}\left(Y_{\text {IV }}\right)$ is compact. The proof is completed.

\section{The Boundedness of Composition Operators}

Theorem 7. If

$$
\begin{aligned}
\frac{\left(1+\left|z z^{T}\right|^{2}-2|z|^{2}-|w|^{2 K}\right)^{q}}{\left(1+\left|z_{2} z_{2}^{T}\right|^{2}-2\left|z_{2}\right|^{2}-\left|w_{2}\right|^{2 K}\right)^{p}}\left|\phi^{\prime}(z, w)\right| & =O(1), \\
\left(z_{2}, w_{2}\right) & \longrightarrow \partial Y_{I V},
\end{aligned}
$$

then $C_{\phi}: \beta^{p}\left(Y_{I V}\right) \rightarrow \beta^{q}\left(Y_{I V}\right)$ is bounded.

Conversely, if $C_{\phi}: \beta^{p}\left(Y_{I V}\right) \rightarrow \beta^{q}\left(Y_{I V}\right)$ is bounded, then

$$
\begin{aligned}
\frac{\left(1+\left|z z^{T}\right|^{2}-2|z|^{2}-|w|^{2 K}\right)^{q} G(z, w)}{\left(1+\left|z_{2} z_{2}^{T}\right|^{2}-2\left|z_{2}\right|^{2}-\left|w_{2}\right|^{2 K}\right)^{p}} & =O(1), \\
\left(z_{2}, w_{2}\right) & \longrightarrow \partial Y_{I V},
\end{aligned}
$$

where $\left(z_{2}, w_{2}\right)=\phi(z, w),(z, w) \in Y_{I V}, \phi=\left(\phi_{1}, \phi_{2}, \ldots\right.$, $\left.\phi_{n}, \phi_{n+1}, \ldots, \phi_{N+n}\right)$, and $K>1$.

$\phi^{\prime}(z, w)$ and $\left|\phi^{\prime}(z, w)\right|^{2}$ see Lemma 1. $G(z, w)=\mid A(z$, $w) \phi^{\prime}(z, w) \mid$

$$
\begin{aligned}
& A(z, w)=\left(2 \overline{z_{2_{1}}}-2 z_{2_{1}} \overline{z_{2} z_{2}^{T}}, \ldots, 2 \overline{z_{2_{n}}}\right. \\
& \left.\quad-2 z_{2_{n}} \overline{z_{2} z_{2}^{T}}, K\left|w_{2}\right|^{2 K-2} \overline{w_{2_{1}}}, \ldots, K\left|w_{2}\right|^{2 K-2} \overline{w_{2_{N}}}\right) .
\end{aligned}
$$

Proof. Let $f \in \beta^{p}\left(Y_{\text {IV }}\right)$. Then,

$$
\begin{aligned}
& |\nabla(f \circ \phi)(z, w)|^{2} \\
& \quad=\sum_{1 \leq \alpha \leq n}\left|\sum_{1 \leq k \leq N+n} \frac{\partial f}{\partial V_{k}}(\phi(z, w)) \frac{\partial \phi_{k}}{\partial z_{\alpha}}(z, w)\right|^{2} \\
& \quad+\sum_{1 \leq \beta \leq N}\left|\sum_{1 \leq k \leq N+n} \frac{\partial f}{\partial V_{k}}(\phi(z, w)) \frac{\partial \phi_{k}}{\partial w_{\beta}}(z, w)\right|^{2} \leq(n \\
& +N) \\
& +\sum_{1 \leq \alpha \leq n}\left(\sum_{1 \leq k \leq N+n}\left|\frac{\partial f}{\partial V_{k}}(\phi(z, w))\right|^{2}\left|\frac{\partial \phi_{k}}{\partial z_{\alpha}}(z, w)\right|^{2}\right) \\
& +(n+N) \sum_{1 \leq \beta \leq N}\left(\sum_{1 \leq k \leq N+n}\left|\frac{\partial f}{\partial V_{k}}(\phi(z, w))\right|^{2}\left|\frac{\partial \phi_{k}}{\partial w_{\beta}}(z, w)\right|^{2}\right) \\
& \quad \leq(n+N)|\nabla f(\phi(z, w))|^{2}\left|\phi^{\prime}(z, w)\right|^{2} .
\end{aligned}
$$


By Lemma 1 and condition (32), there exists a constant $C>0$ such that

$$
\frac{\left(1+\left|z z^{T}\right|^{2}-2|z|^{2}-2|w|^{2 K}\right)^{q}}{\left(1+\left|z_{2} z_{2}^{T}\right|^{2}-2\left|z_{2}\right|^{2}-\left|w_{2}\right|^{2 K}\right)^{p}}\left|\phi^{\prime}(z, w)\right| \leq C
$$

for all $(z, w) \in Y_{\mathrm{IV}}$. So

$$
\begin{aligned}
& {\left[1+\left|z z^{T}\right|^{2}-2|z|^{2}-2|w|^{2 K}\right]^{q}\left|\nabla\left(C_{\phi} f\right)(z, w)\right|} \\
& \leq \sqrt{n+N}\left[1+\left|z z^{T}\right|^{2}-2|z|^{2}-2|w|^{2 K}\right]^{q} \\
& \cdot\left|\phi^{\prime}(z, w)\right||\nabla f(\phi(z, w))| \leq \sqrt{n+N} C\|f\|_{\beta^{p}} \\
& =C^{\prime}\|f\|_{\beta^{p}} .
\end{aligned}
$$

By Lemma 3, we have $f(\phi(0,0)) \leq C^{\prime \prime}\|f\|_{\beta^{p}}$. Thus,

$$
\left\|C_{\phi} f\right\|_{\beta^{q}} \leq C\|f\|_{\beta^{p}} \text {. }
$$

So we get that $C_{\phi}: \beta^{p}\left(Y_{\mathrm{IV}}\right) \rightarrow \beta^{q}\left(Y_{\mathrm{IV}}\right)$ is bounded.

For the conversion, assume that $C_{\phi}: \beta^{p}\left(Y_{\mathrm{IV}}\right) \rightarrow \beta^{q}\left(Y_{\mathrm{IV}}\right)$ is a bounded operator with

$$
\left\|C_{\phi} f\right\|_{\beta^{q}} \leq C\|f\|_{\beta^{p}}
$$

for all $f \in \beta^{p}\left(Y_{\text {IV }}\right)$.

If $p \neq 1 / 2$, we use a family of test functions $\left\{f_{(u, v)}\right.$ : $\left.(u, v) \in Y_{\mathrm{IV}}\right\}$ in $\beta^{p}\left(Y_{\mathrm{IV}}\right)$ which is defined by

$$
\begin{aligned}
& f_{(u, v)}(z, w) \\
& =\frac{1}{2 p-1}\left[\frac{1}{\left(1+z z^{T} \overline{u u^{T}}-2 z \overline{u^{T}}-\langle w, v\rangle^{K}\right)^{2 p-1}}\right. \\
& \quad-1] .
\end{aligned}
$$

Then,

$$
\begin{gathered}
\frac{\partial f_{(u, v)}}{\partial z_{\alpha}}=\frac{1}{2 p-1}(1-2 p) \\
\cdot\left(1+z z^{T} \overline{u u^{T}}-2 z \overline{u^{T}}-\langle w, v\rangle^{K}\right)^{-2 p} \\
\cdot\left(2 z_{\alpha} \overline{u u^{T}}-2 \overline{u_{\alpha}}\right) \\
=\left(1+z z^{T} \overline{u u^{T}}-2 z \overline{u^{T}}-\langle w, v\rangle^{K}\right)^{-2 p} \\
\cdot\left(2 \overline{u_{\alpha}}-2 z_{\alpha} \overline{u u^{T}}\right), \\
\frac{\partial f_{(u, v)}}{\partial w_{\beta}}=\frac{K\langle w, v\rangle^{K-1} \overline{v_{\beta}}}{\left(1+z z^{T} \overline{u u^{T}}-2 z \overline{u^{T}}-\langle w, v\rangle^{K}\right)^{2 p}} .
\end{gathered}
$$

Thus,

$$
\begin{aligned}
& \left|\nabla f_{(u, v)}(z, w)\right|^{2} \\
& =\frac{\sum_{1 \leq \alpha \leq n}\left|2 \overline{u_{\alpha}^{T}}-2 z_{\alpha} \overline{u u^{T}}\right|^{2}+\sum_{1 \leq \beta \leq N}\left|K\langle w, v\rangle^{K-1} \overline{v_{\beta}}\right|^{2}}{\left|1+z z^{T} \overline{u u^{T}}-2 z \overline{u^{T}}-\langle w, v\rangle^{K}\right|^{4 p}} .
\end{aligned}
$$

On one hand, we have

$$
\begin{aligned}
\left(1+\left|z z^{T}\right|^{2}-2|z|^{2}-|w|^{2 K}\right)^{p}\left|\nabla f_{(u, v)}(z, w)\right| & \frac{\left(1+\left|z z^{T}\right|^{2}-2|z|^{2}-|w|^{2 K}\right)^{p}\left(1+\left|u u^{T}\right|^{2}-2|u|^{2}-|v|^{2 K}\right)^{p}}{|| 1+z z^{T} \overline{u u^{T}}-2 z \overline{u^{T}}\left|-(|w||v|)^{K}\right|^{2 p}} \\
\leq & \frac{\left\{\sum_{1 \leq \alpha \leq n}\left|2 \overline{u_{\alpha}^{T}}-2 z_{\alpha} \overline{u u^{T}}\right|^{2}+\sum_{1 \leq \beta \leq N}\left|K\langle w, v\rangle^{K-1} \overline{v_{\beta}}\right|^{2}\right\}^{1 / 2}}{\left(1+\left|u u^{T}\right|^{2}-2|u|^{2}-|v|^{2 K}\right)^{p}} \\
\leq & \frac{\left|2 \bar{u}-2 z \overline{u u^{T}}\right|+K|w|^{K-1}|v|^{K}}{\left(1+\left|u u^{T}\right|^{2}-2|u|^{2}-|v|^{2 K}\right)^{p}} .
\end{aligned}
$$

It is easy to know that

$$
\begin{aligned}
& \left|2 \bar{u}-2 z \overline{u u^{T}}\right| \leq 2|u|+2\left|z \overline{u u^{T}}\right| \leq 4, \\
& K|w|^{K-1}|v|^{K} \leq K .
\end{aligned}
$$

Obviously, $f_{(u, v)}(0,0)=0$. So

$$
\begin{aligned}
& \left\|f_{(u, v)}\right\|_{\beta^{p}}=\left|f_{(u, v)}(0,0)\right| \\
& +\sup _{(z, w) \in Y_{\mathrm{IV}}}\left(1+\left|z z^{T}\right|^{2}-2|z|^{2}-|w|^{2 K}\right)^{p}|\nabla f(z, w)| \\
& \leq \frac{C_{1}}{\left(1+\left|u u^{T}\right|^{2}-2|u|^{2}-|v|^{2 K}\right)^{p}},
\end{aligned}
$$

where $C_{1}=4+K$.

On the other hand, we can get

$$
\begin{gathered}
\left(1+\left|z z^{T}\right|^{2}-2|z|^{2}-|w|^{2 K}\right)^{q}\left|\nabla\left(C_{\phi} f_{(u, v)}\right)(z, w)\right|=(1 \\
\left.+\left|z z^{T}\right|^{2}-2|z|^{2}-|w|^{2 K}\right)^{q} \\
+\left\{\sum_{1 \leq \alpha \leq n}\left|\sum_{1 \leq k \leq N+n} \frac{\partial f_{(u, v)}}{\partial V_{k}}(\phi(z, w)) \frac{\partial \phi_{k}}{\partial z_{\alpha}}(z, w)\right|^{2}\right. \\
\left.+\sum_{1 \leq \beta \leq N}\left|\sum_{1 \leq k \leq N+n} \frac{\partial f_{(u, v)}}{\partial V_{k}}(\phi(z, w)) \frac{\partial \phi_{k}}{\partial w_{\beta}}(z, w)\right|^{2}\right\}^{1 / 2},
\end{gathered}
$$


where

$$
\begin{aligned}
& \sum_{1 \leq k \leq n} \frac{\partial f_{(u, v)}}{\partial V_{k}}(\phi(z, w)) \\
& =\frac{\sum_{1 \leq k \leq n}\left(2 \overline{u_{k}^{T}}-2 z_{2_{k}} \overline{u u^{T}}\right)}{\left(1+z_{2} z_{2}^{T} \overline{u u^{T}}-2 z_{2} \overline{u^{T}}-\left\langle w_{2}, v\right\rangle^{K}\right)^{2 p}}, \\
& \sum_{n+1 \leq k \leq N+n} \frac{\partial f_{(u, v)}}{\partial V_{k}}(\phi(z, w)) \\
& =\frac{\sum_{n+1 \leq k \leq N+n}\left(K\left\langle w_{2}, v\right\rangle^{K-1} \overline{v_{k-n}}\right)}{\left(1+z_{2} z_{2}^{T} \overline{u u^{T}}-2 z_{2} \overline{u^{T}}-\left\langle w_{2}, v\right\rangle^{K}\right)^{2 p}} . \\
& \left(1+\left|z z^{T}\right|^{2}-2|z|^{2}-|w|^{2 K}\right)^{q}\left|\nabla\left(C_{\phi} f_{(u, v)}\right)(z, w)\right| \\
& =\frac{\left(1+\left|z z^{T}\right|^{2}-2|z|^{2}-|w|^{2 K}\right)^{q}}{\left(1+z_{2} z_{2}^{T} \overline{u u^{T}}-2 z_{2} \overline{u^{T}}-\left\langle w_{2}, v\right\rangle^{K}\right)^{2 p}}\left\{\sum_{1 \leq \alpha \leq n} \mid \sum_{1 \leq k \leq n}\left(2 \overline{u_{k}^{T}}-2 z_{2_{k}} \overline{u u^{T}}\right) \frac{\partial \phi_{k}}{\partial z_{\alpha}}(z, w)\right. \\
& +\left.\sum_{n+1 \leq k \leq N+n}\left(K\left\langle w_{2}, v\right\rangle^{K-1} \overline{v_{k-n}}\right) \frac{\partial \phi_{k}}{\partial z_{\alpha}}(z, w)\right|^{2}+\sum_{1 \leq \beta \leq N} \mid \sum_{1 \leq k \leq n}\left(2 \overline{u_{k}^{T}}-2 z_{2_{k}} \overline{u u^{T}}\right) \frac{\partial \phi_{k}}{\partial w_{\beta}}(z, w) \\
& \left.+\left.\sum_{n+1 \leq k \leq N+n}\left(K\left\langle w_{2}, v\right\rangle^{K-1} \overline{v_{k-n}}\right) \frac{\partial \phi_{k}}{\partial w_{\beta}}(z, w)\right|^{2}\right\}^{1 / 2}
\end{aligned}
$$

Set $(u, v)=\left(z_{2}, w_{2}\right)=\phi(z, w)$; by (39), we have

$$
\begin{aligned}
& \frac{\left(1+\left|z z^{T}\right|^{2}-2|z|^{2}-|w|^{2 K}\right)^{q}}{\left(1+z_{2} z_{2}^{T} \overline{u u^{T}}-2 z_{2} \overline{u^{T}}-\left\langle w_{2}, v\right\rangle^{K}\right)^{2 p}}\left\{\sum_{1 \leq \alpha \leq n} \mid \sum_{1 \leq k \leq n}\left(2 \overline{u_{k}^{T}}-2 z_{2_{k}} \overline{u u^{T}}\right) \frac{\partial \phi_{k}}{\partial z_{\alpha}}(z, w)\right. \\
& +\left.\sum_{n+1 \leq k \leq N+n}\left(K\left\langle w_{2}, v\right\rangle^{K-1} \overline{v_{k-n}}\right) \frac{\partial \phi_{k}}{\partial z_{\alpha}}(z, w)\right|^{2}+\sum_{1 \leq \beta \leq N} \mid \sum_{1 \leq k \leq n}\left(2 \overline{u_{k}^{T}}-2 z_{2_{k}} \overline{u u^{T}}\right) \frac{\partial \phi_{k}}{\partial w_{\beta}}(z, w) \\
& \left.+\left.\sum_{n+1 \leq k \leq N+n}\left(K\left\langle w_{2}, v\right\rangle^{K-1} \overline{v_{k-n}}\right) \frac{\partial \phi_{k}}{\partial w_{\beta}}(z, w)\right|^{2}\right\}^{1 / 2} \leq C \frac{C_{1}}{\left(1+\left|z_{2} z_{2}^{T}\right|^{2}-2\left|z_{2}\right|^{2}-\left|w_{2}\right|^{2 K}\right)^{p}} .
\end{aligned}
$$

Furthermore, we have

$$
\begin{aligned}
& \qquad \frac{\left(1+\left|z z^{T}\right|^{2}-2|z|^{2}-|w|^{2 K}\right)^{q}}{\left(1+\left|z_{2} z_{2}^{T}\right|^{2}-2\left|z_{2}\right|^{2}-\left|w_{2}\right|^{2 K}\right)^{p}} G(z, w) \leq C_{2} . \\
& \text { If } p=1 / 2 \text {, set }
\end{aligned}
$$$$
f_{(u, v)}(z, w)=\ln \frac{1}{\left(1+z z^{T} \overline{u u^{T}}-2 z \overline{u^{T}}-\langle w, v\rangle^{K}\right)} .
$$

Then,

$$
\begin{aligned}
& \frac{\partial f_{(u, v)}}{\partial z_{\alpha}}=\frac{2 \overline{u_{\alpha}^{T}}-2 z_{\alpha} \overline{u u^{T}}}{\left(1+z z^{T} \overline{u u^{T}}-2 z \overline{u^{T}}-\langle w, v\rangle^{K}\right)}, \\
& \frac{\partial f_{(u, v)}}{\partial w_{\beta}}=\frac{K\langle w, v\rangle^{K-1} \overline{v_{\beta}}}{\left(1+z z^{T} \overline{u u^{T}}-2 z \overline{u^{T}}-\langle w, v\rangle^{K}\right)} .
\end{aligned}
$$

For the same reason, it can be proved that (33) holds. The details are omitted here. The proof is completed. 


\section{The Compactness of Composition Operators}

Theorem 8. If

$$
\begin{aligned}
\frac{\left(1+\left|z z^{T}\right|^{2}-2|z|^{2}-|w|^{2 K}\right)^{q}}{\left(1+\left|z_{2} z_{2}^{T}\right|^{2}-2\left|z_{2}\right|^{2}-\left|w_{2}\right|^{2 K}\right)^{p}}\left|\phi^{\prime}(z, w)\right| & =o(1), \\
\left(z_{2}, w_{2}\right) & \longrightarrow \partial Y_{I V},
\end{aligned}
$$

then $C_{\phi}: \beta^{p}\left(Y_{I V}\right) \rightarrow \beta^{q}\left(Y_{I V}\right)$ is compact.

Conversely, if $C_{\phi}: \beta^{p}\left(Y_{I V}\right) \rightarrow \beta^{q}\left(Y_{I V}\right)$ is compact, then

$$
\begin{aligned}
& \frac{\left(1+\left|z z^{T}\right|^{2}-2|z|^{2}-|w|^{2 K}\right)^{q} G(z, w)}{\left(1+\left|z_{2} z_{2}^{T}\right|^{2}-2\left|z_{2}\right|^{2}-\left|w_{2}\right|^{2 K}\right)^{p}}=o(1), \\
&\left(z_{2}, w_{2}\right) \longrightarrow \partial Y_{I V}
\end{aligned}
$$

where $\left(z_{2}, w_{2}\right)=\phi(z, w),(z, w) \in Y_{I V}, \phi=\left(\phi_{1}, \phi_{2}, \ldots\right.$, $\left.\phi_{n}, \phi_{n+1}, \ldots, \phi_{N+n}\right), K>1,\left|\phi^{\prime}(z, w)\right|$, and $G(z, w)$; see Theorem 7 .

Proof. Let $\left\{f_{v}\right\}$ be a bounded sequence in $\beta^{p}\left(Y_{\text {IV }}\right)$ with $\left\|f_{v}\right\|_{\beta^{p}} \leq C$ and $f_{v} \rightarrow 0$ uniformly on compact subsets of $Y_{\mathrm{IV}}$. Furthermore, by Weierstrass Theorem, it is easy to show that $\left\{\nabla f_{v}\right\} \rightarrow 0$ uniformly on compact subsets of $Y_{\mathrm{IV}}$. Thus, for any $\varepsilon>0$, there exists $\delta>0$ such that

$$
\frac{\left(1+\left|z z^{T}\right|^{2}-2|z|^{2}-|w|^{2 k}\right)^{q}}{\left(1+\left|z_{2} z_{2}^{T}\right|^{2}-2\left|z_{2}\right|^{2}-\left|w_{2}\right|^{2 k}\right)^{p}}\left|\phi^{\prime}(z, w)\right|<\varepsilon
$$

whenever $\operatorname{dist}\left(\left(z_{2}, w_{2}\right), \partial Y_{\text {IV }}\right)<\delta$. Then,

$$
\begin{gathered}
\left(1+\left|z z^{T}\right|^{2}-2|z|^{2}-|w|^{2 K}\right)^{q}\left|\nabla\left(C_{\phi} f_{v}\right)(z, w)\right| \\
\leq \sqrt{n+N}\left(1+\left|z z^{T}\right|^{2}-2|z|^{2}-|w|^{2 K}\right)^{q} \\
\cdot\left|\nabla f_{v}(\phi(z, w))\right|\left|\phi^{\prime}(z, w)\right| \leq \sqrt{n+N} \varepsilon\left\|f_{v}\right\|_{\beta^{p}} .
\end{gathered}
$$

Write $E_{\delta}=\left\{\left(z_{2}, w_{2}\right) \in Y_{\mathrm{IV}}, \operatorname{dist}\left(\left(z_{2}, w_{2}\right), \partial Y_{\mathrm{IV}}\right) \geq \delta\right\}$, and then $E_{\delta}$ is a compact subset of $Y_{\text {IV }}$.

Let $\left(z_{2}, w_{2}\right) \in E_{\delta}$; then, there exists a constant $M \in(0,1)$ such that

$$
\frac{1}{\left(1+\left|z_{2} z_{2}^{T}\right|^{2}-2\left|z_{2}\right|^{2}-\left|w_{2}\right|^{2 K}\right)^{p}} \leq \frac{1}{M^{p}}<+\infty .
$$

Therefore, there exists a constant $C$ such that

$$
\frac{\left(1+\left|z z^{T}\right|^{2}-2|z|^{2}-|w|^{2 K}\right)^{q}}{\left(1+\left|z_{2} z_{2}^{T}\right|^{2}-2\left|z_{2}\right|^{2}-\left|w_{2}\right|^{2 K}\right)^{p}}\left|\phi^{\prime}(z, w)\right| \leq C .
$$

So

$$
\begin{aligned}
& \left(1+\left|z z^{T}\right|^{2}-2|z|^{2}-|w|^{2 K}\right)^{q}\left|\nabla\left(C_{\phi} f_{v}\right)(z, w)\right| \\
& \quad \leq C \sqrt{n+N}\left|\nabla f_{v}(\phi(z, w))\right| .
\end{aligned}
$$

Since $f_{v}(\phi(0,0)) \rightarrow 0$ as $v \rightarrow \infty$, from (56) and (59) we obtain

$$
\begin{aligned}
& \left\|C_{\phi} f_{v}\right\|_{\beta^{q}}=\left|f_{v}(\phi(0,0))\right| \\
& \quad+\sup \left(1+\left|z z^{T}\right|^{2}-2|z|^{2}-|w|^{2 K}\right)^{q} \\
& \cdot\left|\nabla\left(f_{v} \circ \phi\right)(z, w)\right| \longrightarrow 0, \quad v \longrightarrow \infty .
\end{aligned}
$$

By Lemma 6 , we know that $C_{\phi}: \beta^{p}\left(Y_{\text {IV }}\right) \rightarrow \beta^{q}\left(Y_{\text {IV }}\right)$ is compact.

For the conversion, assume that (54) fails; then, there exists a sequence $\left\{\left(z^{j}, w^{j}\right)\right\}$ in $Y_{\text {IV }}$ with $\phi\left(z^{j}, w^{j}\right) \rightarrow \partial Y_{\text {IV }}$ as $j \rightarrow \infty$ and $\varepsilon_{0}>0$ such that

$$
\frac{\left(1+\left|z^{j} z^{j^{T}}\right|^{2}-2\left|z^{j}\right|^{2}-\left|w^{j}\right|^{2 K}\right)^{q} G\left(z^{j}, w^{j}\right)}{\left(1+\left|z_{2}^{j} z_{2}^{j^{T}}\right|^{2}-2\left|z_{2}^{j}\right|^{2}-\left|w_{2}^{j}\right|^{2 K}\right)^{p}} \geq \varepsilon_{0}
$$

for all $j=1,2, \ldots$.

We will construct a family of functions $\left\{f_{j}(z, w)\right\}$ satisfying the following three conditions:

(I) $\left\{f_{j}(z, w)\right\}$ is a bounded sequence in $\beta^{p}\left(Y_{\text {IV }}\right)$;

(II) $\left\{f_{j}(z, w)\right\}$ tends to zero uniformly on compact subsets of $Y_{\mathrm{IV}}$

(III) $\left\|C_{\phi} f_{j}(z, w)\right\|_{\beta^{q}} \nrightarrow 0, j \rightarrow \infty$.

This contradicts with the compactness of $C_{\phi}$. Hence, we prove that (54) is necessary for that $C_{\phi}: \beta^{p}\left(Y_{\mathrm{IV}}\right) \rightarrow \beta^{q}\left(Y_{\mathrm{IV}}\right)$ being compact.

If $p \neq 1 / 2$, set

$$
\begin{aligned}
& f_{j}(z, w) \\
& \left.=\frac{1}{2 p-1} \frac{\left(1+\left|z_{2}^{j} z_{2}^{j^{T}}\right|^{2}-2\left|z_{2}^{j}\right|^{2}-\left|w_{2}^{j}\right|^{2 K}\right)^{p}}{\left(1+z z^{T} z_{2}^{j} z_{2}^{j^{T}}\right.}-2 z z_{2}^{j^{T}}-\left\langle w, w_{2}^{j}\right\rangle^{K}\right)^{2 p-1}
\end{aligned}
$$

We have 


$$
\begin{aligned}
& \left(1+\left|z z^{T}\right|^{2}-2|z|^{2}-|w|^{2 K}\right)^{p}\left|\nabla f_{j}(z, w)\right|=\left(1+\left|z z^{T}\right|^{2}-2|z|^{2}-|w|^{2 K}\right)^{p}\left(1+\left|z_{2}^{j} z_{2}^{j^{T}}\right|^{2}-2\left|z_{2}^{j}\right|^{2}-\left|w_{2}^{j}\right|^{2 K}\right)^{p} \\
& \frac{\left\{\sum_{1 \leq \alpha \leq n}\left|2 \overline{z_{2_{\alpha}}^{j^{T}}}-2 z_{\alpha} \overline{z_{2}^{j} z_{2}^{j^{T}}}\right|^{2}+K^{2} \sum_{1 \leq \beta \leq N}\left(\left|\left\langle w, w_{2}^{j}\right\rangle\right|^{2 K-2}\left|\overline{w_{2_{\beta}}^{j}}\right|^{2}\right)\right\}^{1 / 2}}{\left|1+z z^{T} \overline{z_{2}^{j} z_{2}^{j^{T}}}-2 z \overline{z_{2}^{j^{T}}}-\left\langle w, w_{2}^{j}\right\rangle^{K}\right|^{2 p}} \\
& \leq \frac{\left(1+\left|z z^{T}\right|^{2}-2|z|^{2}-|w|^{2 K}\right)^{p}\left(1+\left|z_{2}^{j} z_{2}^{j^{T}}\right|^{2}-2\left|z_{2}^{j}\right|^{2}-\left|w_{2}^{j}\right|^{2 K}\right)^{p}}{\| 1+z z^{T} \overline{z_{2}^{j} z_{2}^{j^{T}}}-2 z \overline{z_{2}^{j^{T}}}\left|-\left(|w|\left|w_{2}^{j}\right|\right)^{K}\right|^{2 p}} \\
& \cdot\left(2\left|\overline{z_{2}^{j^{T}}}-z \overline{z_{2}^{j} z_{2}^{j^{T}}}\right|+K|w|^{K-1}\left|w_{2}^{j}\right|^{K}\right) \text {. }
\end{aligned}
$$

It is easy to know that

$$
\begin{aligned}
\left|\overline{z_{2}^{j^{T}}}-\overline{z z_{2}^{j} z_{2}^{j^{T}}}\right| & \leq\left|\overline{z_{2}^{j^{T}}}\right|+\left|z \overline{z_{2}^{j} z_{2}^{j^{T}}}\right| \leq 2, \\
|K||w|^{K-1}\left|w_{2}^{j}\right|^{K} & \leq K .
\end{aligned}
$$

Combining with Lemma 5, we can get $\left(1+\left|z z^{T}\right|^{2}-2|z|^{2}-\right.$ $\left.|w|^{2 K}\right)^{p}\left|\nabla f_{j}(z, w)\right| \leq C_{1}$, where $C_{1}=4+K$. Thus, $\left\{f_{j}(z, w)\right\}$ is a bounded sequence in $\beta^{p}\left(Y_{\mathrm{IV}}\right)$. This means that $\left\{f_{j}(z, w)\right\}$ satisfies condition (I).

By Lemma 5,

$$
\begin{aligned}
\left|f_{j}(z, w)\right| \leq \frac{1}{2 p-1} \frac{\left|\left(1+\left|z_{2}^{j} z_{2}^{j^{T}}\right|^{2}-2\left|z_{2}^{j}\right|^{2}-\left|w_{2}^{j}\right|^{2 K}\right)^{p}\right|}{\left(1+\left|z z^{T}\right|^{2}-2|z|^{2}-|w|^{2 K}\right)^{p-1 / 2}\left(1+\left|z_{2}^{j} z_{2}^{j^{T}}\right|^{2}-2\left|z_{2}^{j}\right|^{2}-\left|w_{2}^{j}\right|^{2 K}\right)^{p-1 / 2}} \\
=\frac{1}{2 p-1} \frac{\left|\left(1+\left|z_{2}^{j} z_{2}^{j^{T}}\right|^{2}-2\left|z_{2}^{j}\right|^{2}-\left|w_{2}^{j}\right|^{2 K}\right)^{1 / 2}\right|}{\left(1+\left|z z^{T}\right|^{2}-2|z|^{2}-|w|^{2 K}\right)^{p-1 / 2}} .
\end{aligned}
$$

When $\left(z_{2}^{j}, w_{2}^{j}\right) \rightarrow \partial Y_{\text {IV }}$ as $j \rightarrow \infty$, then $\left(1+\left|z_{2}^{j} z_{2}^{j^{T}}\right|^{2}-2\left|z_{2}^{j}\right|^{2}-\right.$ $\left.\left|w_{2}^{j}\right|^{2 K}\right) \rightarrow 0$. Since $(z, w) \in H$ and $H$ is a compact subset of $Y_{\mathrm{IV}}$, then $\inf \left(1+\left|z z^{T}\right|^{2}-2|z|^{2}-|w|^{2 K}\right)>0$. So $f_{j}(z, w) \rightarrow 0$ as $j \rightarrow \infty$ uniformly on compact subsets of $Y_{\mathrm{IV}}$. This means that $\left\{f_{j}(z, w)\right\}$ satisfies condition (II)

$$
\begin{aligned}
\left(1+\left|z^{j} z^{j^{T}}\right|^{2}-2\left|z^{j}\right|^{2}-\left|w^{j}\right|^{2 K}\right)^{q}\left|\nabla\left(C_{\phi} f_{j}\right)\left(z^{j}, w^{j}\right)\right| & \\
& =\frac{\left(1+\left|z^{j} z^{j^{T}}\right|^{2}-2\left|z^{j}\right|^{2}-\left|w^{j}\right|^{2 K}\right)^{q}}{\left(1+\left|z_{2}^{j} z_{2}^{j^{T}}\right|^{2}-2\left|z_{2}^{j}\right|^{2}-\left|w_{2}^{j}\right|^{2 K}\right)^{p}}\left\{\sum_{1 \leq \alpha \leq n} \mid \sum_{1 \leq k \leq n}\left(2 \overline{z_{2_{k}}^{j}}-2 z_{2_{k}}^{j} \overline{z_{2}^{j} z_{2}^{j^{T}}}\right) \frac{\partial \phi_{k}}{\partial z_{\alpha}}\left(z^{j}, w^{j}\right)\right. \\
& +\left.\sum_{n+1 \leq k \leq n+N}\left(K\left|w_{2}^{j}\right|^{2 K-2} \overline{w_{2_{k-n}^{j}}}\right) \frac{\partial \phi_{k}}{\partial z_{\alpha}}\left(z^{j}, w^{j}\right)\right|^{2}+\sum_{1 \leq \beta \leq N} \mid \sum_{1 \leq k \leq n}\left(2 \overline{z_{2_{k}}^{j}}-2 z_{2_{k}} \overline{z_{2}^{j} z_{2}^{j^{T}}}\right) \frac{\partial \phi_{k}}{\partial w_{\beta}^{j}}\left(z^{j}, w^{j}\right) \\
& \left.+\left.\sum_{n+1 \leq k \leq n+N}\left(K\left|w_{2}^{j}\right|^{2 K-2} \overline{w_{2_{k-n}^{j}}^{j}}\right) \frac{\partial \phi_{k}}{\partial w_{\beta}}\left(z^{j}, w^{j}\right)\right|^{2}\right\}^{1 / 2}=\frac{\left(1+\left|z^{j} z^{j^{T}}\right|^{2}-2\left|z^{j}\right|^{2}-\left|w^{j}\right|^{2 K}\right)^{q} G\left(z^{j}, w^{j}\right)}{\left(1+\left|z_{2}^{j} z_{2}^{j^{T}}\right|^{2}-2\left|z_{2}^{j}\right|^{2}-\left|w_{2}^{j}\right|^{2 K}\right)^{p}} .
\end{aligned}
$$


Condition (61) shows that $\left\|C_{\phi} f_{j}\right\| \rightarrow 0$ as $j \rightarrow+\infty$ and $\left\{f_{j}(z, w)\right\}$ satisfies condition (III).

When $p=1 / 2$, set

$$
\begin{gathered}
f_{j}(z, w)=\left(1+\left|z_{2}^{j} z_{2}^{j^{T}}\right|^{2}-2\left|z_{2}^{j^{T}}\right|^{2}-\left|w_{2}^{j}\right|^{2 K}\right)^{1 / 2} \\
\cdot \ln \left(1+z z^{T} \overline{z_{2}^{j} z_{2}^{j^{T}}}-2 z \overline{z_{2}^{j^{T}}}-\left\langle w, w_{2}^{j}\right\rangle^{K}\right),
\end{gathered}
$$

$$
\begin{aligned}
(1+ & \left.\left|z^{j} z^{j^{T}}\right|^{2}-2\left|z^{j}\right|^{2}-\left|w^{j}\right|^{2 K}\right)^{q}\left|\nabla\left(C_{\phi} f_{j}\right)\left(z^{j}, w^{j}\right)\right| \\
& =\frac{\left(1+\left|z^{j} z^{j^{T}}\right|^{2}-2\left|z^{j}\right|^{2}-\left|w^{j}\right|^{2 K}\right)^{q}}{\left(1+\left|z_{2}^{j} z_{2}^{j^{T}}\right|^{2}-2\left|z_{2}^{j}\right|^{2}-\left|w_{2}^{j}\right|^{2 K}\right)^{1 / 2}}\left\{\sum_{1 \leq \alpha \leq n} \mid \sum_{1 \leq k \leq n}\left(2 \overline{z_{2_{k}}^{j}}-2 z_{2_{k}}^{j} \overline{z_{2}^{j} z_{2}^{j^{T}}}\right) \frac{\partial \phi_{k}}{\partial z_{\alpha}^{j}}\left(z^{j}, w^{j}\right)\right. \\
& +\left.\sum_{n+1 \leq k \leq n+N}\left(K\left|w_{2}^{j}\right|^{2 K-2} \overline{w_{2_{k-n}^{j}}^{j}}\right) \frac{\partial \phi_{k}}{\partial z_{\alpha}^{j}}\left(z^{j}, w^{j}\right)\right|^{2}+\sum_{1 \leq \beta \leq N} \mid \sum_{1 \leq k \leq n}\left(2 \overline{z_{2_{k}^{j}}}-2 z_{2_{k}}^{j} \overline{z_{2}^{j} z_{2}^{j^{T}}}\right) \frac{\partial \phi_{k}}{\partial w_{\beta}^{j}}\left(z^{j}, w^{j}\right) \\
& \left.+\left.\sum_{n+1 \leq k \leq n+N}\left(K\left|w_{2}^{j}\right|^{2 K-2} \overline{w_{2_{k-n}^{j}}^{j}}\right) \frac{\partial \phi_{k}}{\partial w_{\beta}^{j}}\left(z^{j}, w^{j}\right)\right|^{2}\right\}^{1 / 2}=\frac{\left(1+\left|z^{j} z^{j^{T}}\right|^{2}-2\left|z^{j}\right|^{2}-\left|w^{j}\right|^{2 K}\right)^{q} G\left(z^{j}, w^{j}\right)}{\left(1+\left|z_{2}^{j} z_{2}^{j^{T}}\right|^{2}-2\left|z_{2}^{j}\right|^{2}-\left|w_{2}^{j}\right|^{2 K}\right)^{p}} .
\end{aligned}
$$

for all $j=1,2, \ldots$. Then,

$$
\begin{aligned}
& \frac{\partial f_{j}}{\partial z_{\alpha}} \\
& =\frac{\left(1+\left|z_{2}^{j} z_{2}^{j^{T}}\right|^{2}-2\left|z_{2}^{j}\right|^{2}-\left|w_{2}^{j}\right|^{2 K}\right)^{1 / 2}\left(2 z_{\alpha} \overline{z_{2}^{j} z_{2}^{j^{T}}}-2 \overline{z_{2_{\alpha}}^{j^{T}}}\right)}{\left(1+z z^{T} \overline{z_{2}^{j} z_{2}^{j^{T}}}-2 z \overline{z_{2}^{j^{T}}}-\left\langle w, w_{2}^{j}\right\rangle^{K}\right)} \\
& \frac{\partial f_{j}}{\partial w_{\beta}} \\
& =\frac{\left(1+\left|z_{2}^{j} z_{2}^{j^{T}}\right|^{2}-2\left|z_{2}^{j}\right|^{2}-\left|w_{2}^{j}\right|^{2 K}\right)^{1 / 2}\left(-K\left\langle w, w_{2}^{j}\right\rangle^{K-1} \overline{w_{2_{\beta}}^{j}}\right)}{\left(1+z z^{T} \overline{z_{2}^{j} z_{2}^{j^{T}}}-2 z \overline{z_{2}^{j^{T}}}-\left\langle w, w_{2}^{j}\right\rangle^{K}\right)} .
\end{aligned}
$$

in $\beta^{p}\left(Y_{\text {IV }}\right)$ and tends to zero uniformly on compact subsets of $Y_{\mathrm{IV}}$. By (68),
From the assumption, we get $\left(1+\left|z^{j} z^{j^{T}}\right|^{2}-2\left|z^{j}\right|^{2}-\right.$ $\left.\left|w^{j}\right|^{2 K}\right)^{q}\left|\nabla\left(C_{\phi} f_{j}\right)\left(z^{j}, w^{j}\right)\right| \geq \varepsilon_{0}$. This means that $\left\{f_{j}\right\}$ satisfies condition (III). The proof is completed.

\section{Conflict of Interests}

The authors declare that there is no conflict of interests regarding the publication of this paper.

\section{Acknowledgments}

The authors thank the editor and the referee for their valuable comments and suggestions. This research is funded by the National Natural Science Foundation of China (Grant no. 11171285).

\section{References}

[1] K. Madigan and A. Matheson, "Compact composition operators on the Bloch space," Transactions of the American Mathematical Society, vol. 347, no. 7, pp. 2679-2687, 1995. 
[8] R. M. Timoney, "Bloch functions in several complex variables," The Bulletin of the London Mathematical Society, vol. 12, no. 4, pp. 241-267, 1980.

[9] Z. H. Zhou and J. H. Shi, "Compactness of composition operators on the Bloch space in classical bounded symmetric domains," The Michigan Mathematical Journal, vol. 50, no. 2, pp. 381-405, 2002.

[10] W. P. Yin, "The Bergman kernels on super-cartan domains of the first type," Science in China Series A: Mathematics, vol. 43, no. 1, pp. 13-21, 2000.

[11] Q. K. Lu, The Classical Manifolds and the Classical Domains, Shanghai Scientific and Technical Publisher, Shanghai, China, 1963 (Chinese).

[12] L. K. Hua, "An Inequality on determinant," Acta Mathematica Sinica, vol. 5, pp. 463-470, 1955.

[13] J. B. Su, X. X. Miao, and H. J. Li, "Generalizations of Hua's inequalities and an application," Journal of Mathematical Inequalities, vol. 9, no. 1, pp. 27-45, 2015. 


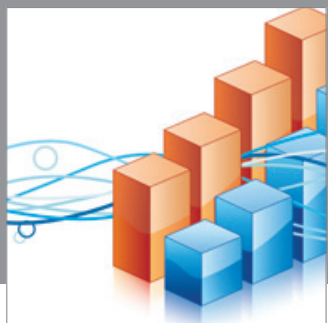

Advances in

Operations Research

mansans

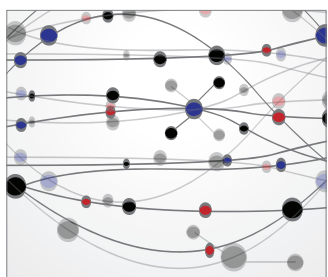

The Scientific World Journal
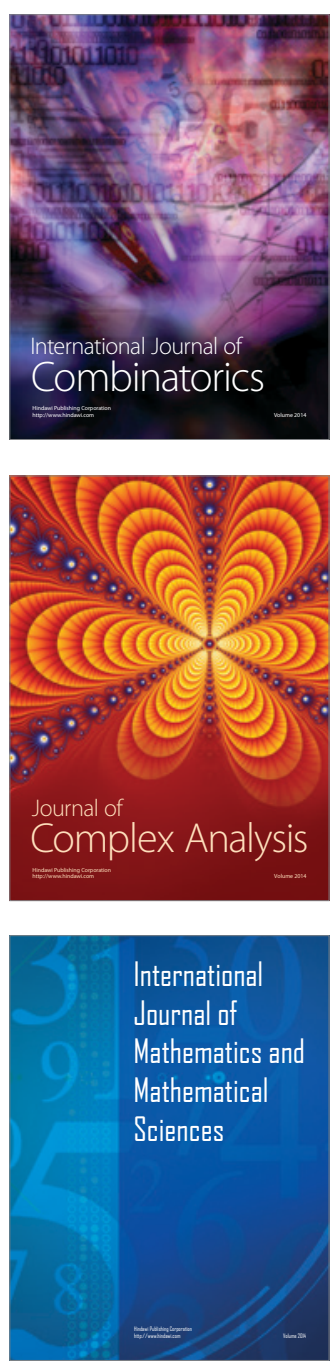
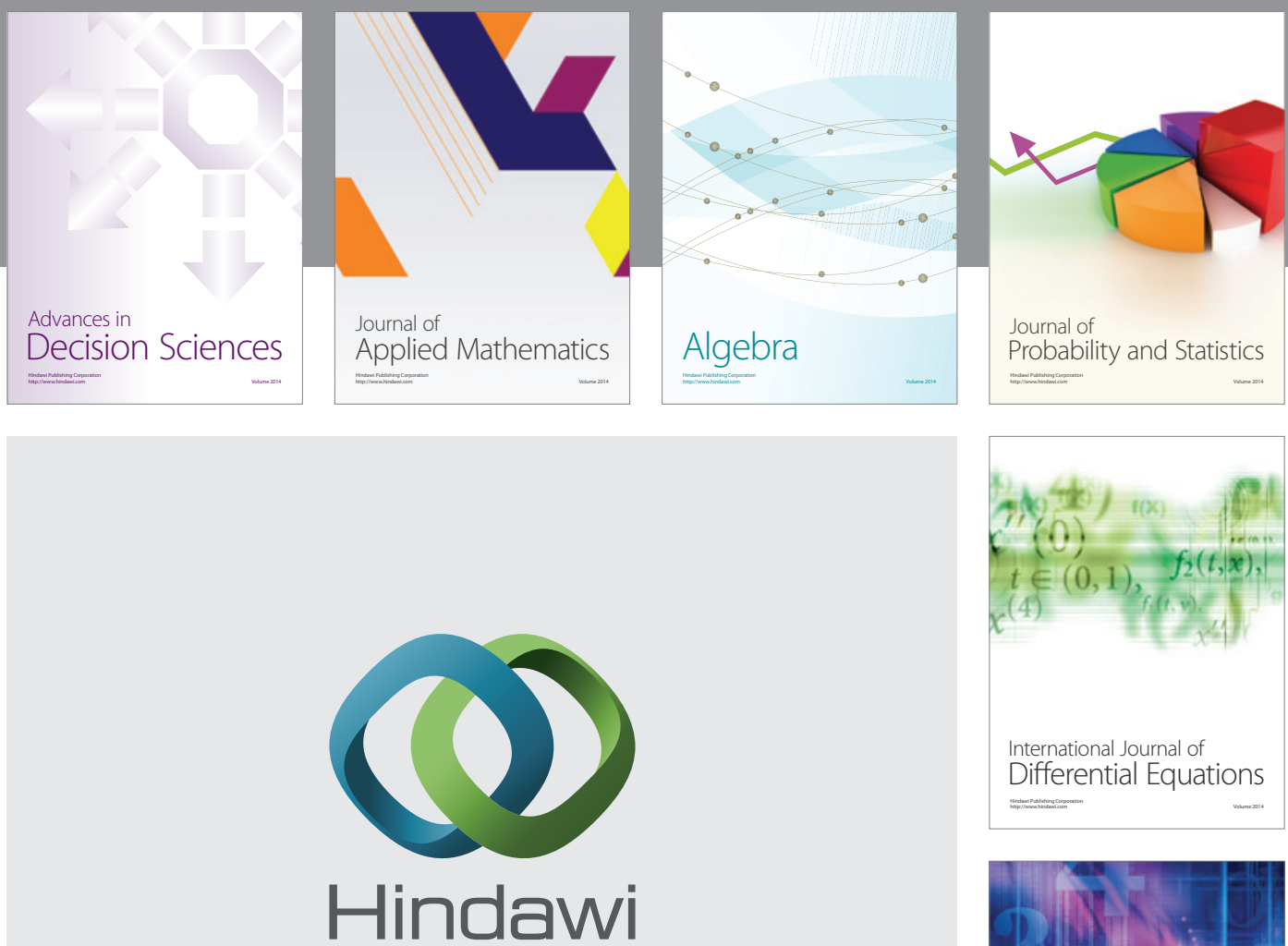

Submit your manuscripts at http://www.hindawi.com
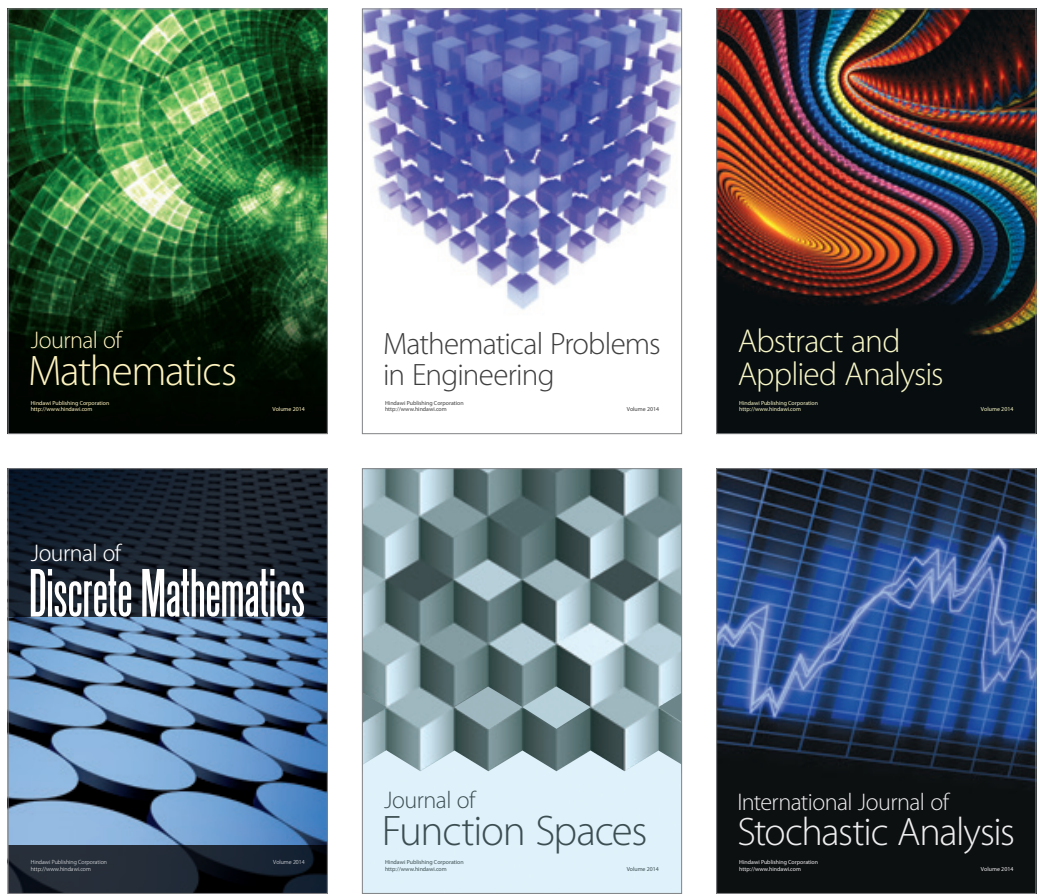

Journal of

Function Spaces

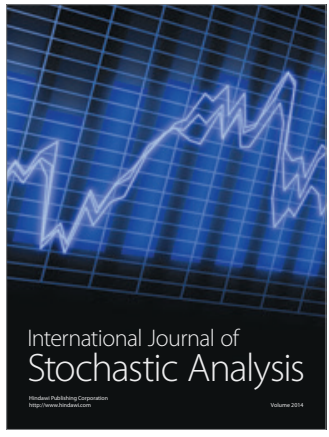

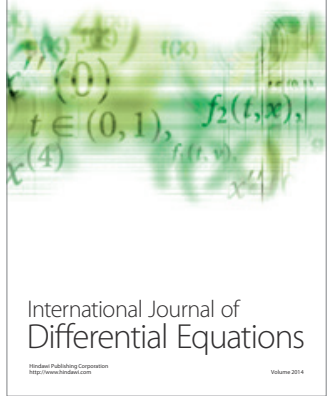
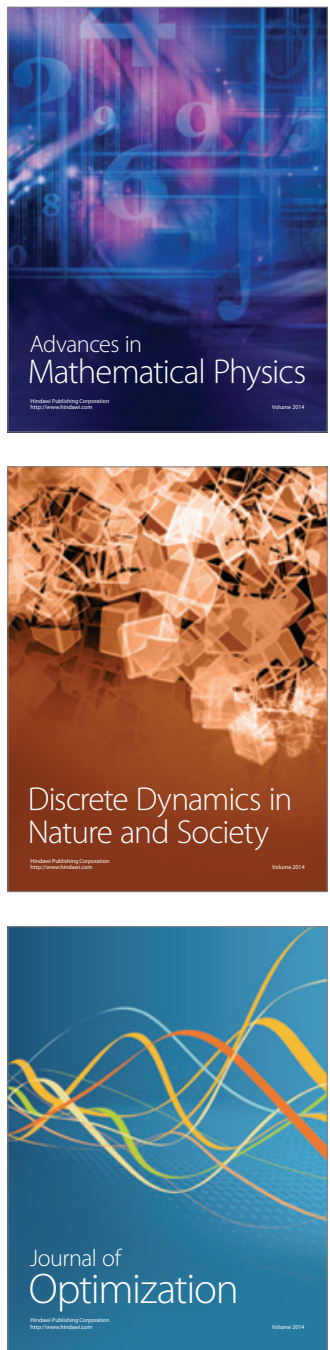